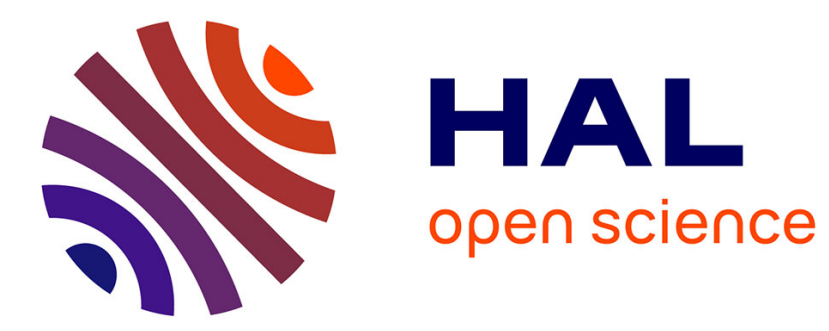

\title{
Polaritonic modes in a dense cloud of cold atoms
}

N. J Schilder, Christophe Sauvan, J.-P. Hugonin, Stephan Jennewein, Yvan R.

P. Sortais, Antoine Browaeys, J.-J. Greffet

\section{To cite this version:}

N. J Schilder, Christophe Sauvan, J.-P. Hugonin, Stephan Jennewein, Yvan R. P. Sortais, et al.. Polaritonic modes in a dense cloud of cold atoms. Physical Review A : Atomic, molecular, and optical physics [1990-2015], 2016, 93 (6), pp.063835. 10.1103/PhysRevA.93.063835 . hal-01643446

\section{HAL Id: hal-01643446 \\ https://hal.science/hal-01643446}

Submitted on 21 Nov 2017

HAL is a multi-disciplinary open access archive for the deposit and dissemination of scientific research documents, whether they are published or not. The documents may come from teaching and research institutions in France or abroad, or from public or private research centers.
L'archive ouverte pluridisciplinaire HAL, est destinée au dépôt et à la diffusion de documents scientifiques de niveau recherche, publiés ou non, émanant des établissements d'enseignement et de recherche français ou étrangers, des laboratoires publics ou privés. 


\title{
Polaritonic modes in a dense cloud of cold atoms
}

\author{
N. J. Schilder, ${ }^{*}$ C. Sauvan, J.-P. Hugonin, S. Jennewein, Y. R. P. Sortais, A. Browaeys, and J.-J. Greffet \\ Laboratoire Charles Fabry, Institut d'Optique Graduate School, CNRS, Université Paris-Saclay, 91127 Palaiseau Cedex, France
}

(Received 21 October 2015; revised manuscript received 18 April 2016; published 20 June 2016)

\begin{abstract}
We analyze resonant light scattering by a cloud of cold atoms with randomly distributed positions in a regime where resonant dipole-dipole interactions between scatterers cannot be neglected. Using a microscopic approach we calculate numerically the collective eigenmodes of the cloud for many realizations. It is found that there always exists a small number of polaritonic modes. Using a macroscopic approach, we show that the atomic cloud is equivalent to a homogeneous particle with an effective permittivity and that there is a one-to-one correspondence between the microscopic polaritonic modes and the modes of the homogeneous particle.
\end{abstract}

DOI: 10.1103/PhysRevA.93.063835

\section{INTRODUCTION}

Light scattering by an ensemble of particles is usually viewed as a multiple scattering process involving a sequence of single scattering events. Each scatterer is considered to be in the far field of the others so that a scattering matrix approach can be used to describe each scattering event [1]. When the density of the system increases, correlations and near-field resonant dipole-dipole interactions between scatterers start playing a role. These effects and also coherence effects can be included using a coherent theory of multiple scattering [2-5]. Within this framework, it is possible to show that the electric field averaged over an ensemble of realizations of the system obeys a propagation equation in a homogeneous system with an effective dielectric permittivity. Many standard models are available to derive the effective permittivity in the dilute regime $[3,6-8]$. The regime where recurrent scattering, i.e., the electromagnetic field gets scattered at least twice by the same scatterer, becomes significant remains largely unexplored experimentally.

The physics of multiple scattering of light has been revisited in the context of resonant atomic gases in the last fifteen years. Backscattering enhancement has been measured in the low-intensity regime where a classical description of scattering is valid $[9,10]$. Cold atoms allow changing the scattering regime by detuning the laser: from optically thin to optically thick media [11] and from dilute to dense media [12]. In the dense regime, novel effects are expected as pointed out by Morice et al. [13]. The recurrent scattering has been studied theoretically in Refs. [14,15]. These works predict that resonant dipole-dipole interaction becomes significant when the dipole-dipole interaction strength parameter $\rho / k^{3}$ is larger than unity, where $\rho$ is the number of atoms per unit volume and $\lambda_{0}=2 \pi / k$ is the atomic resonance wavelength. It is interesting to note that this condition can be cast in the form $k l<1$ where $l \sim k^{2} / \rho$ is the elastic mean free path on resonance. This condition is thus similar to the so-called IoffeRegel criterion of Anderson localization [3]. Furthermore, in this density regime, it has been shown that the effective dielectric permittivity can be negative [16]. The decrease of the elastic mean free path due to resonant dipole-dipole interaction has been discussed in Refs. [17-19]. Another interesting aspect of scattering by a dense cold-atom system is the presence

${ }^{*}$ Corresponding author: nick.schilder@institutoptique.fr of collective effects. Recently, single-photon superradiance has been studied both theoretically [20] and experimentally [21-24]. Bienaimé et al. described light scattering quantum optically for a finite-size system and found subradiant and superradiant states [25]. They predicted that superradiance gives rise to directional light scattering. It has also been reported that spontaneous emission is dominated by superradiant modes and that the emission spectrum displays both negative and positive Lamb shifts in the presence of resonant dipole-dipole interactions [26]. Sokolov et al. [27] analyzed the response of an ensemble of cold resonant scatterers in a spherical volume and they compared a modal microscopic description with a macroscopic description based on the effective permittivity in the mean field approximation; both approaches are in good agreement for low densities. In contexts such as random lasing, light localization, and subradiance, microscopic modes of a system of interacting atoms in the scalar approximation have been studied [25,26,28-31].

In this work, we study dense $\left(\rho / k^{3} \gtrsim 1\right)$ and disordered systems consisting of $N$ resonant dipoles in a small volume with dimensions comparable to the resonant wavelength [see Fig. 1(a)]. We consider cold atoms in vacuum with a negligible Doppler effect. We study light scattering by this system using, on one hand, a microscopic model accounting for all interactions between atoms and, on the other hand, a macroscopic model based on a homogeneous system with an effective permittivity [see Fig. 1(b)]. The effective permittivity is then derived numerically by fitting the scattered intensity averaged over an ensemble of 300 different realizations of the system. This allows us to compare both the eigenfrequencies and the spatial structures of the eigenmodes of both microscopic and macroscopic systems.

As already pointed out in Ref. [25], superradiant modes are responsible for coherent light scattering. In this work, we observe that some of them have the following properties: (i) all atoms contribute to the mode, (ii) the frequency and the spatial structure of the mode is independent of the realization but depends on the geometry and density of the system so that they are very robust against disorder. Hence, despite the fact that the system is disordered, these modes are analogous to the polaritons introduced by Hopfield to analyze collective excitations in condensed matter for ordered systems [32]. We thus refer to these superradiant modes as polaritonic modes. We further observe that the spatial structure of these modes coincides with the spatial structure of the modes of the effective homogeneous particle with effective permittivity. We thus 
conclude that there is a one-to-one correspondence between modes of a particle with effective permittivity and polaritonic modes in an atomic system.

The analysis developed in this work is not limited to cold-atom systems but is also applicable to condensed-matter systems of coupled oscillators such as an ensemble of quantum dots and layers of organic molecules with electronic transitions or vibrations in a crystal. Let us give two examples where the polaritonic behavior survives the introduction of disorder and can be described in the framework of an effective permittivity. As a first example, we consider surface phonon polaritons. A phonon is a collective excitation which results from the interaction potential between neighbors. For optical phonons, these collective mechanical excitations are coupled to the electromagnetic field, resulting in a polariton [33]. These approaches can be applied to many different crystals such as $\mathrm{SiC}$ or quartz. It is now interesting to consider amorphous glass. There, the concept of phonon disappears as there is no periodicity of the system. However, it turns out that the permittivity still has a negative real part so that surface phonon polaritons are still predicted by classical electrodynamics and indeed observed experimentally. This raises the question of the underlying microscopic excitation.

The second example is the optical behavior of a disordered ensemble of densely packed quantum dots or organic molecules such as $\mathbf{J}$ aggregates. It has been observed that layers of these resonant materials deposited on metallic films display strong coupling with surface plasmons [34-41]. The experimental results can be explained using a resonant effective permittivity for the ensemble scatterers. It has further been observed that the modes are spatially coherent. The previous examples share the basic physical ingredients with our model consisting of $N$ cold atoms in a cloud. They are dense and disordered systems of $N$ resonant scatterers.

The paper is organized as follows. First, using a microscopic model, we study the collective eigenmodes of the system consisting of $N$ cold atoms in a small volume as depicted in Fig. 1(a). We find that some of them, called hereafter

(a)
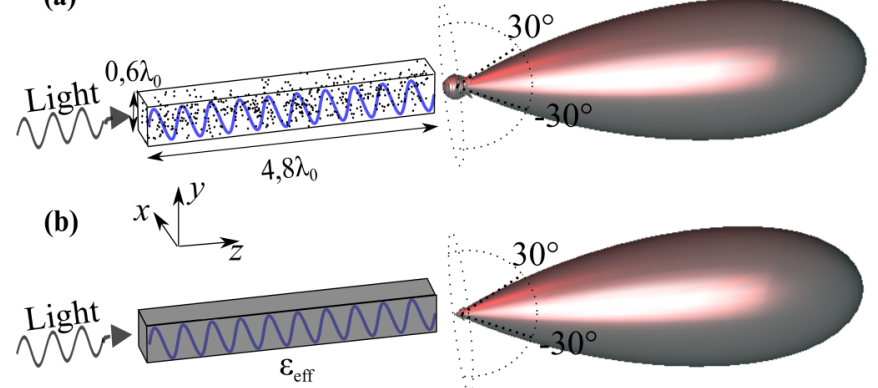

FIG. 1. (a) System under study: $N=450$ atoms uniformly distributed in a rectangular box. The atomic density is $\rho=k^{3}$. The incoming light excites the collective eigenmodes of the system (visualized by the blue sinusoid), which gives rise to a scattering pattern in, essentially, the forward direction. Here, we have plotted the modulus square of the electric field radiated in the far field at resonance, averaged over 1500 realizations, $\left\langle|\mathbf{E}|^{2}\right\rangle$. (b) Homogeneous medium described by an effective permittivity $\varepsilon_{\text {eff }}$ exhibiting optical resonances. The scattering pattern, $|\mathbf{E}|^{2}$, is found to be similar to (a) except for the diffuse light, which is by definition null in (b). polaritonic, are robust against disorder, involve all atoms, and are superradiant. We then analyze light scattering by the system using this model and compare it with an effective permittivity as sketched in Fig. 1(b). We show that polaritonic modes can be identified with the macroscopic optical modes of that system. We also discuss the origin of losses in the effective permittivity.

\section{MODEL}

We consider a cloud of atoms uniformly distributed in a rectangular box [see Fig. 1(a)]. The dipoles, being cold atoms in vacuum, experience no nonradiative losses and a negligible Doppler effect with respect to their radiative linewidth $\Gamma_{0}$. In the numerical simulations presented below, we have taken rubidium- 87 atoms, $\lambda_{0}=780 \mathrm{~nm}$, and $\Gamma_{0}=$ $2 \pi \times(6 \mathrm{MHz})$. The number of atoms, $N=450$, and the dimensions of the box, $4.8 \lambda_{0} \times 0.6 \lambda_{0} \times 0.6 \lambda_{0}$, correspond to typical experimental conditions obtained by laser cooling and trapping techniques in wavelength-size optical dipole traps [12]. With such parameters, $\rho / k^{3} \sim 1$, so recurrent scattering starts playing a significant role. We also show some results for a rectangular cloud with dimensions $2.1 \lambda_{0} \times 0.4 \lambda_{0} \times 0.4 \lambda_{0}$ and density $\rho / k^{3} \sim 5$. The cloud is investigated in the weakexcitation limit, where its optical properties can be described by classical optics [26,28]. Because the system is dense, we treat the electromagnetic field vectorially to properly take into account the $1 / r^{3}$ dependence of the near-field interactions ( $r$ is the interatomic distance). The atoms are modeled as pointlike and identical scatterers characterized by an isotropic electric polarizability matrix $\overline{\bar{\alpha}}(\omega)=\alpha(\omega) \mathbb{1}$, where $\alpha(\omega)=\left(3 \pi \Gamma_{0} / k^{3}\right) /\left(\omega_{0}-\omega-i \Gamma_{0} / 2\right)$, with $\omega_{0}=c k$ being the transition frequency and $c$ being the speed of light in vacuum. This polarizability model corresponds to a classical $J=0 \rightarrow J=1$ atom with three transitions. It assumes elastic scattering events and therefore no nonradiative decay channels.

The microscopic eigenmodes of the atomic cloud are obtained by searching for a self-consistent solution of the coupled-dipole equations in the absence of a driving electric field. The induced dipoles $\mathbf{p}_{i}$ can be written as $\mathbf{p}_{i}=$ $\varepsilon_{0} \overline{\bar{\alpha}}(\omega) \sum_{j \neq i} \mathbf{E}_{j \rightarrow i}\left(\mathbf{r}_{i}\right)$, where $\mathbf{E}_{j \rightarrow i}\left(\mathbf{r}_{i}\right)=\mu_{0} \omega^{2} \overline{\bar{G}}\left(\mathbf{r}_{j}, \mathbf{r}_{i} ; \omega\right) \mathbf{p}_{j}$ is the electric field at the position of scatterer $i$ created by the induced dipole of scatterer $j$. Here, $\overline{\bar{G}}\left(\mathbf{r}_{j}, \mathbf{r}_{i} ; \omega\right)$ is the vacuum Green tensor [42]. The dipoles are thus coupled through the following linear system of equations:

$$
\mathbf{p}_{i}-\frac{\omega^{2}}{c^{2}} \alpha(\omega) \sum_{j \neq i} \overline{\bar{G}}\left(\mathbf{r}_{j}, \mathbf{r}_{i}, \omega\right) \mathbf{p}_{j}=0 .
$$

Assuming $\omega^{2} \overline{\bar{G}}\left(\mathbf{r}_{j}, \mathbf{r}_{i}, \omega\right) \approx \omega_{0}^{2} \overline{\bar{G}}\left(\mathbf{r}_{j}, \mathbf{r}_{i}, \omega_{0}\right)$ turns this problem into an eigenvalue problem. Because of the narrow atomic linewidth and the nonresonant character of the vacuum Green tensor, this is an accurate assumption. The eigenvalue problem is of the form $\overline{\bar{A}}\left(\omega_{0}\right) \mathbf{P}_{\beta}=\left(\omega_{\beta}-i \frac{\Gamma_{\beta}}{2}\right) \mathbf{P}_{\beta}$, where the matrix

$$
\overline{\bar{A}}\left(\omega_{0}\right)=\left(\begin{array}{ccc}
\left(\omega_{0}-i \frac{\Gamma_{0}}{2}\right) \mathbb{I} & \frac{-3 \pi c \Gamma_{0}}{\omega_{0}} \overline{\bar{G}}_{2 \rightarrow 1} & \ldots \\
\frac{-3 \pi c \Gamma_{0}}{\omega_{0}} \overline{\bar{G}}_{1 \rightarrow 2} & \left(\omega_{0}-i \frac{\Gamma_{0}}{2}\right) \mathbb{I} & \\
\vdots & & \ddots
\end{array}\right),
$$

of which all submatrices are of size $3 \times 3$. 
This eigenvalue problem has $3 N$ eigenvalues. The corresponding eigenvectors $\mathbf{P}_{\beta}$ are composed of the vector components of all $N$ dipole moments, $\mathbf{P}_{\beta}=$ $\left[p_{1 x}^{\beta}, p_{1 y}^{\beta}, p_{1 z}^{\beta}, \ldots p_{N x}^{\beta}, p_{N y}^{\beta}, p_{N z}^{\beta}\right]^{\top}$. They are normalized such that $\left|\mathbf{P}_{\beta}\right|^{2}=\sum_{j}\left|\mathbf{p}_{j}^{\beta}\right|^{2}=1$.

\section{EIGENSPECTRUM ANALYSIS}

Figure 2 shows all 405000 eigenfrequencies in the complex plane obtained from 300 realizations of the cloud. We observe three distinct families of modes, each defined in terms of their collective linewidth $\Gamma_{\beta}$ and their collective frequency shift $\Omega_{\beta}=\omega_{\beta}-\omega_{0}$ : (1) $\Gamma_{\beta} \in\left\{0,2 \Gamma_{0}\right\}$ and $\left|\Omega_{\beta}\right| \gg \Gamma_{0}$, (2) $\Gamma_{\beta} \gg \Gamma_{0}$, and (3) others. Type 1 eigenmodes have a collective linewidth of either $\Gamma \approx 2 \Gamma_{0}$ or $\Gamma \ll \Gamma_{0}$ and a large frequency shift. Figure 3(a) shows a typical eigenmode of type 1. It contains only two excited atoms, so we call it a dimer mode. When two dipoles oscillate in phase and are very close together, the electric fields of both dipoles interfere constructively. Therefore, the pair of dipoles radiates twice as fast $\left(\Gamma=2 \Gamma_{0}\right)$ as they would independently. However, when the dipoles oscillate out of phase, their electric fields interfere destructively, and the pair of dipoles is strongly subradiant $\left(\Gamma \ll \Gamma_{0}\right)$. The large level splitting is due to the large resonant dipole-dipole interaction.

Within the type 2 family of modes, one can distinguish a few patches (four in these cases) of modes (see rectangles in the insets of Fig. 2), which we denote as polaritonic modes for reasons explained below. More precisely, for a given realization, the spectrum of eigenfrequencies always contains the same number of polaritonic modes, with each mode falling
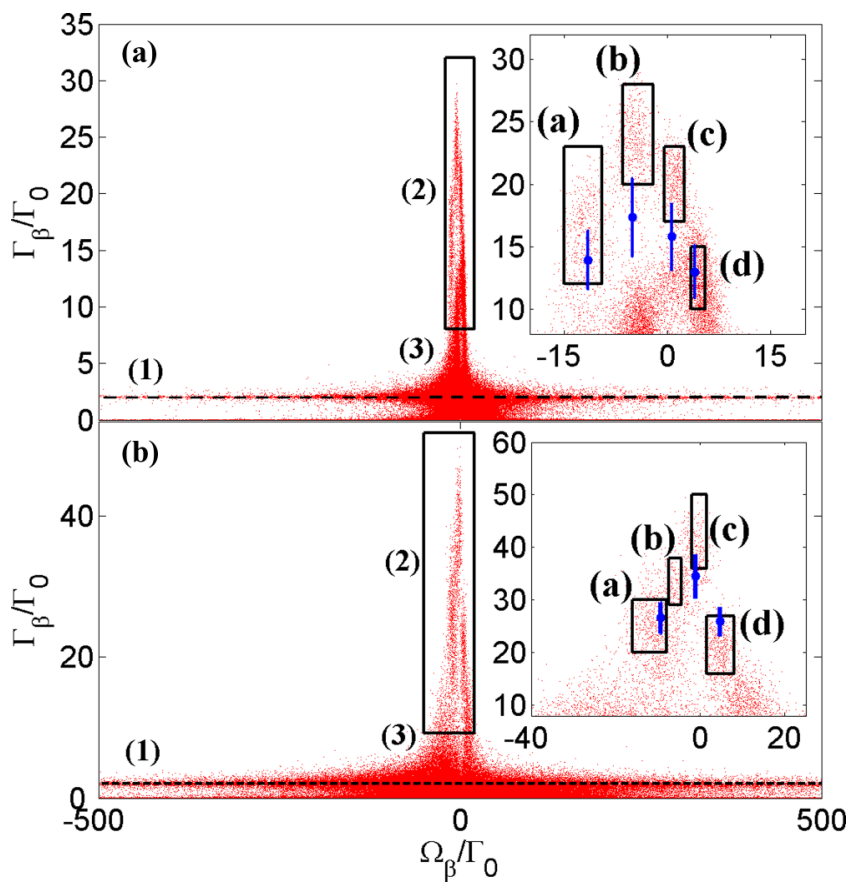

FIG. 2. The three types of eigenmodes are indicated by the numbers (1) through (3). The inset shows a closeup of the boxed part of the main figure, where the polaritonic modes (a), (b), (c), and (d) for both (a) $\rho / k^{3}=1$ and (b) $\rho / k^{3}=5$ are visualized in Fig. 7. (a)
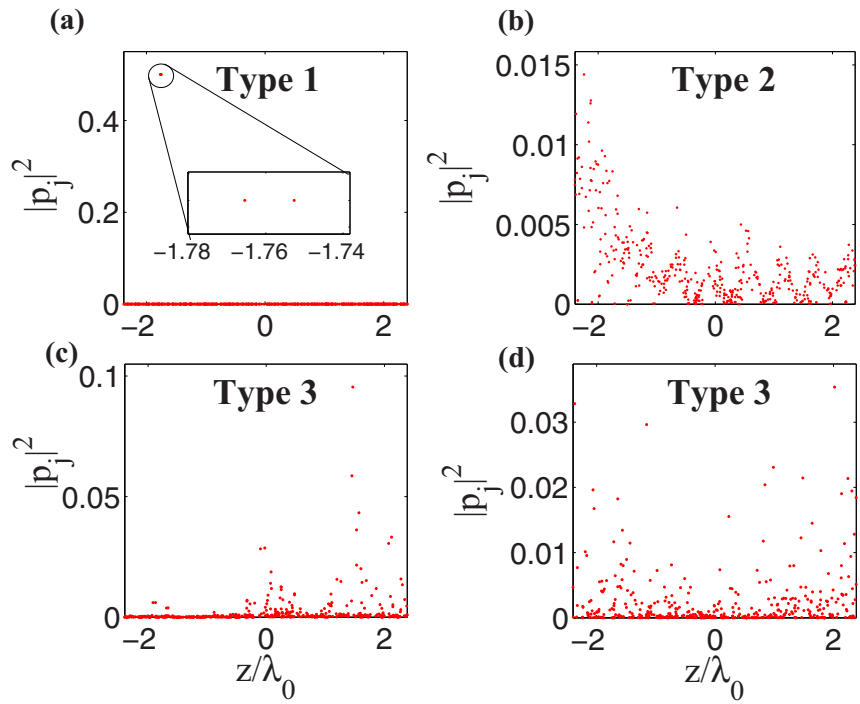

FIG. 3. Typical eigenmodes for the cloud with $\rho / k=1$ are shown. (a) Type 1 eigenmodes are called dimer modes. The dipoles are very close to each other. (b) A polaritonic mode shows an oscillatory behavior. (c) Bunches of dipoles oscillate. (d) Many dipoles oscillate, but without the spatial oscillatory behavior, so there is no periodic arrangement of the dipoles.

into one of these patches [43]. For a dense atomic system, in the scalar approximation, these patches have also been obtained numerically by Goetschy et al. [30,31]. Figure 3(b) shows a plot of the dipole moments squared $\left|\mathbf{p}_{j}\right|^{2}$ for a typical mode of type 2 . For this collective mode, most of the atoms are excited and Fig. 3(b) suggests $\left|\mathbf{p}_{j}\right|^{2}$ oscillates spatially along the $z$ axis. We have checked that this oscillation is an intrinsic property of the homogeneous system, i.e., that it is a property of the shape and density of the sample but not of the exact positions of the atoms. To do this, we calculate the average dipole moment squared $\left\langle|\mathbf{p}|^{2}\right\rangle$, where $\langle\cdots\rangle$ denotes averaging both inside thin slices perpendicular to the long axis of the box, and over 300 realizations of the uniform atomic distribution. Here, only the modes inside rectangle (a) in the inset of Fig. 2(a) are taken into account in the average. The rectangles are taken such that they contain most of the polaritonic modes of a specific kind. Figure 4(b) shows that the spatial oscillation observed in Fig. 4(a) survives the ensemble averaging. Note that the symmetry of the macroscopic mode structure with respect to the plane $z=0$ is restored by the averaging procedure. Their spatial structure is the same for all realizations: It depends on the density and shape of the cloud. These facts confirm that these few type 2 eigenmodes are polaritonic by nature. This is in contrast with the other types of modes, which do not show this spatial periodicity along the $z$ axis nor involve all the dipoles simultaneously.

All modes we have not discussed so far belong to type 3. These modes involve either many dipoles distributed throughout the whole volume but without a regular spatial structure [Fig. 3(d)], as the one observed in Fig. 3(b), or a localized mode involving only a subset of dipoles [Fig. 3(c)]. In order to demonstrate that neither type 1 nor type 3 modes have a spatial structure, as opposed to polaritonic modes, we have calculated the average dipole moment square along the 


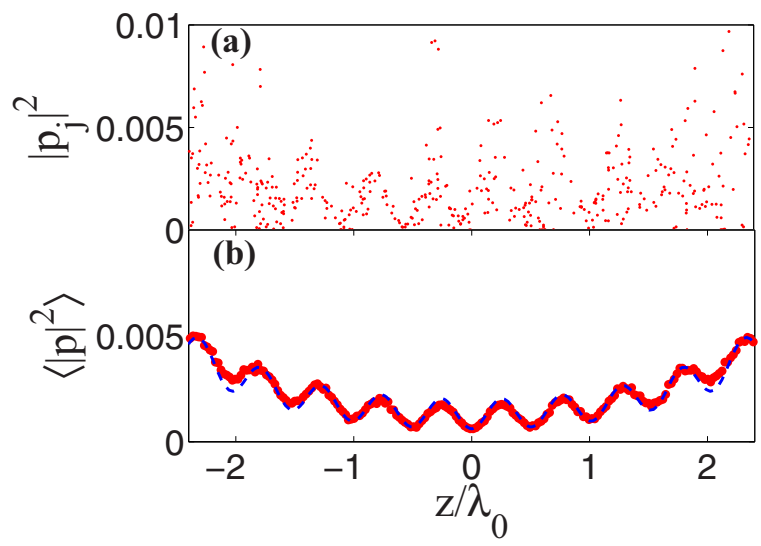

FIG. 4. (a) Spatial structure of a typical microscopic eigenmode of type 2 for $\rho / k^{3}=1$. The dipole moment squared $\left|\mathbf{p}_{j}\right|^{2}$ of each atom $j$ has been plotted, with the atoms being sorted by their $z$ coordinate. (b) Average dipole moments squared $\left\langle|\mathbf{p}|^{2}\right\rangle$ (red dots). The ensemble average is performed over the polaritonic modes inside the black solid rectangle (a) in the inset of Fig. 2(a). The macroscopic mode of the homogeneous cloud (blue solid line) coincides very well with the average polaritonic mode, taking into account a small offset due to the fluctuations of the dipoles amplitudes from one realization to another. Refer to the appendix for a discussion about this offset.

long axis of the box for these modes. It is seen in Fig. 5 that these modes do not possess the oscillatory structure observed for type 2 modes.

The periodic arrangement of the dipole moments, which only exists for the type 2 eigenmodes, allows phase matching of the radiation by the dipoles and therefore single-photon superradiance along the axis of the object. Note that this phase matching only occurs within a rather limited solid angle $(\sim 2 \pi / 15)$. This is why the linewidth is not enhanced by a factor $N$ as is the case for superradiance by $N$ emitters in a subwavelength volume. As the other modes do not possess such a spatial arrangement, the collective linewidth for both type 1 and 3 modes is smaller.
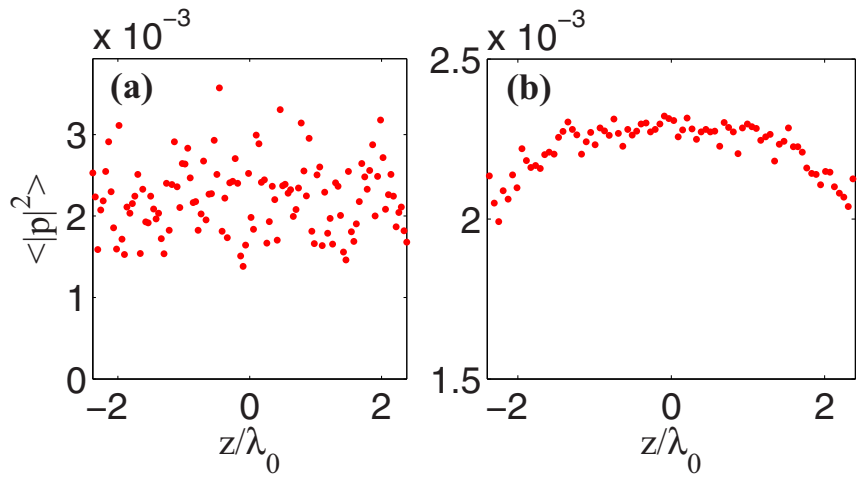

FIG. 5. The average dipole moment square of type 1 (a) and type 3 (b) eigenmodes along the long axis of the box do not show any spatial oscillatory structure, like type 2 does. (a) All eigenmodes with $\left|\Omega_{\beta}\right|>150 \Gamma_{0}$ have been taken into account for the averaging. (b) All modes within the region defined by $\left|\Omega_{\beta}\right|<19$ and $\Gamma_{\beta}<3 \Gamma_{0}$ have been taken into account for the averaging. The density of the cloud is $\rho / k^{3}=1$.

\section{LIGHT SCATTERING}

We now study light scattering by the cloud of atoms. Solving the coupled-dipoles equations using the laser field as an external driving source allows us to calculate the scattered field $\mathbf{E}$ in the far field for a given realization of the cloud when it is illuminated by an $x$-polarized plane wave, as is done in our recent work [44]. After averaging over 1500 realizations, the scattering pattern obtained near resonance $\left(\omega=\omega_{0}\right)$ exhibits one lobe in the forward direction [see Fig. 1(a)] and some diffuse light which is on average quasi-isotropic and has a smaller amplitude. However, these two features have comparable contributions when integrated over the full solid angle with $54 \%$ and $46 \%$ of the total scattered light, respectively. The lobe in the forward direction is very similar to the diffraction pattern originating from a homogeneous particle, suggesting that one could replace the cloud, with its random graininess, by a homogeneous cloud with the same shape and extract an effective permittivity.

We understand these observations by decomposing the electric field $\mathbf{E}$ scattered by the random medium into an ensemble-averaged field $\langle\mathbf{E}\rangle$ (also denoted coherent) and a fluctuating field $\delta \mathbf{E}$ (also denoted incoherent), $\mathbf{E}=\langle\mathbf{E}\rangle+\delta \mathbf{E}$, with $\langle\delta \mathbf{E}\rangle=0[2,4]$. $\langle\mathbf{E}\rangle$ satisfies the Helmholtz equation in an effective homogeneous medium with an effective permittivity $\varepsilon_{\text {eff: }}: \nabla^{2}\langle\mathbf{E}\rangle+\frac{\omega^{2}}{c^{2}} \varepsilon_{\text {eff }}\langle\mathbf{E}\rangle=0[2,4]$. From this it follows that $\langle\mathbf{E}\rangle$ is the field diffracted by an effective medium with effective permittivity $\varepsilon_{\text {eff }}$ and with the same shape as the cloud. The scattered intensity can also be decomposed in a coherent and incoherent contribution $\left\langle|\mathbf{E}|^{2}\right\rangle=|\langle\mathbf{E}\rangle|^{2}+$ $\left\langle|\delta \mathbf{E}|^{2}\right\rangle \propto I_{\text {coh }}+I_{\text {incoh. }}$. The first term corresponds to the lobe in the forward direction in Fig. 1(a), while the second term is the quasi-isotropic diffuse light.

\section{EFFECTIVE PERMITTIVITY}

We now proceed to the extraction of the effective permittivity of the cloud as a function of frequency. Textbook meanfield theory gives the Lorentz-Lorenz relation between the macroscopic permittivity of a medium and the polarizability of the scatterers

$$
\frac{\varepsilon_{\mathrm{LL}}(\omega)-1}{\varepsilon_{\mathrm{LL}}(\omega)+2}=\frac{\rho \alpha(\omega)}{3} .
$$

This formula takes only partially dipole-dipole interactions into account by the local-field correction [45]. Recurrent scattering is not included, while its impact on the effective properties is expected to be of importance for dense systems $[4,19,46]$. In order to derive the effective permittivity by accounting for multiple scattering, we solve an inverse problem. We compare the coherent contribution $|\langle\mathbf{E}\rangle|^{2}$ of the far-field scattering pattern of the atomic cloud with that of an effective homogeneous particle with a permittivity $\varepsilon_{\text {eff }}$ and the same geometry as the cloud. The latter is numerically calculated with an aperiodic Fourier modal method (a-FMM) [47], using $\varepsilon_{\text {eff }}$ as a fitting parameter. We find that the far-field scattering pattern computed using either the effective homogeneous particle or the ensemble average microscopic cloud agree within $1 \%$ [compare Figs. 1(a) and 1(b)], showing that the effective permittivity approach is valid. We conclude that this 
macroscopic approach captures both the recurrent scattering and the collective effects of the microscopic picture. By repeating this procedure for different frequency detunings $\delta \omega=\omega-\omega_{0}$, we obtain the spectrum of the effective permittivity (see Fig. 6), which is found to be significantly different from the prediction by Eq. (2). Our results evidence that the Lorentz-Lorenz formula is not valid for a dense cloud of resonant scatterers; the discrepancy between the simulation and the Lorentz-Lorenz theory increases with the density, showing that the dipole-dipole interactions become stronger $[46,48]$. This result has been recently studied experimentally using a cloud of cold atoms with similar shape and density [44]. It is all the more remarkable as the system is five orders of magnitude more dilute than a gas at ambiant conditions for which the Lorentz-Lorenz theory is an accurate description.

It is noteworthy that at resonance the gas is described by a permittivity with a negative real part and thus optically behaves as a metal. From the permittivity we derive the imaginary part of the refractive index $n_{\text {eff }}=\varepsilon_{\text {eff }}^{1 / 2}$ and thus find the mean free path at resonance, $l=1 /\left[k \operatorname{Im}\left(n_{\mathrm{eff}}\right)\right]$. We find $l=1 /(1.5 k)$, indicating that the Ioffe-Regel criterion is satisfied [3]. Yet, we have found many modes extended throughout the whole system, indicating that the localization length, if any, is larger than the size of the system.

\section{CONNECTION BETWEEN POLARITONIC MODES AND MACROSCOPIC MODES}

We have seen that the far-field scattering pattern of the atomic cloud is very similar to that of an effective homogeneous particle with an effective permittivity. We now make explicit the relation between the (macroscopic) modes of the homogeneous particle and the (microscopic) polaritonic modes. To do that, we use the fitted effective permittivity $\varepsilon_{\text {eff }}(\omega)$ (see solid lines in Fig. 6) and calculate the macroscopic modes of the homogeneous particle, which are poles of the scattering matrix, by iteratively solving Maxwell's equations in the complex frequency plane [49]. Remarkably, we find

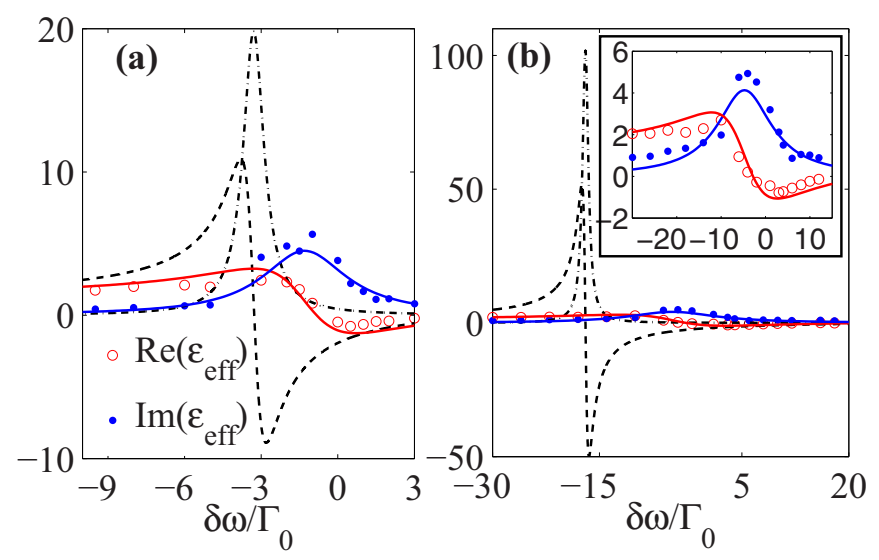

FIG. 6. Effective permittivity $\varepsilon_{\text {eff }}(\omega)$ for (a) $\rho / k^{3}=1$, and (b) $\rho / k^{3}=5$. The real and imaginary parts of the effective permittivity (circles and dots) largely differ from the predictions of LorentzLorenz theory (dashed curves). Solid lines: Lorentzian fit of the numerical data. (a) $\times 10^{-3}$

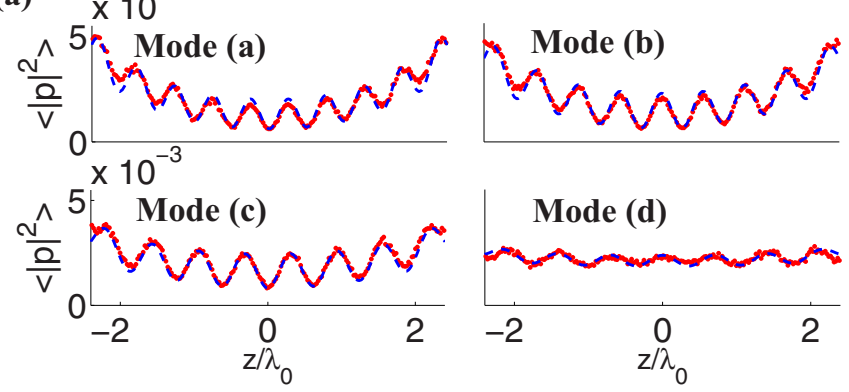

(b)
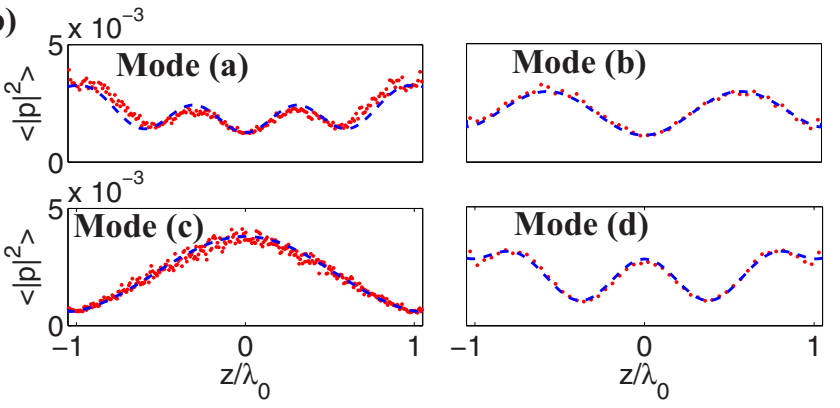

FIG. 7. The average dipole moment squared of polaritonic modes (red dots) is plotted along the long axis of the rectangular box. Superimposed (blue dashed curve) is $|\mathbf{E}|^{2}$ of the macroscopic mode of the homogeneous object. Each panel belongs to a different polaritonic mode. Refer to Fig. 2 for the eigenfrequencies of the modes. For the averaging procedure, we divided the system along its long axis in thin slices. The dipole moment squared is averaged per slice and then averaged over 300 realizations. (a) $\rho / k^{3}=1$ and (b) $\rho / k^{3}=5$.

that the macroscopic modes coincide (within error bars) with the above-mentioned polaritonic modes (see Fig. 2). Despite the fact that the geometrical length of the cloud is fixed and the frequency of the laser is almost fixed (close to $\omega_{0}$ ), it is possible to find several longitudinal modes because of the strong dispersion of the medium close to resonance. This provides a physical explanation of the results reported by $\mathrm{Li}$ et al. [26], who studied the spontaneous emission spectrum of a dense cloud of atoms and found blue- and red-shifted modes.

Figure 4(b) shows that the above-mentioned coincidence is not accidental: The microscopic and macroscopic modes have not only the same frequency and linewidth, but also the same spatial structure along the $z$ axis. Figure 7 shows the spatial agreement for all polaritonic modes for both $\rho / k^{3}=1$ and $\rho / k^{3}=5$. This illustrates that the coincidence is not restricted to a particular value of the interaction strength parameter $\rho / k^{3}$. The same agreement holds for the transverse dimensions of the box. Figure 8 shows an example of this agreement. We observe that the number of oscillations as observed in Fig. 7 decreases by one from the leftmost patch to the rightmost patch in Fig. 7(a). The same holds for the polaritonic modes in Fig. 7(b), taking into account that modes (b) and (c) actually have a different polarization as the other modes. Modes (b) and (c) are polarized along the long axis of the box ( $z$ axis), whereas the other modes are transversely polarized, i.e., in the $(x, y)$ plane. Modes (a)-(d) for $\rho / k^{3}=1$ are also mostly transversally polarized. Our analysis thus sheds new light on the connection between the microscopic and macroscopic approaches of scattering, as one can directly identify the 


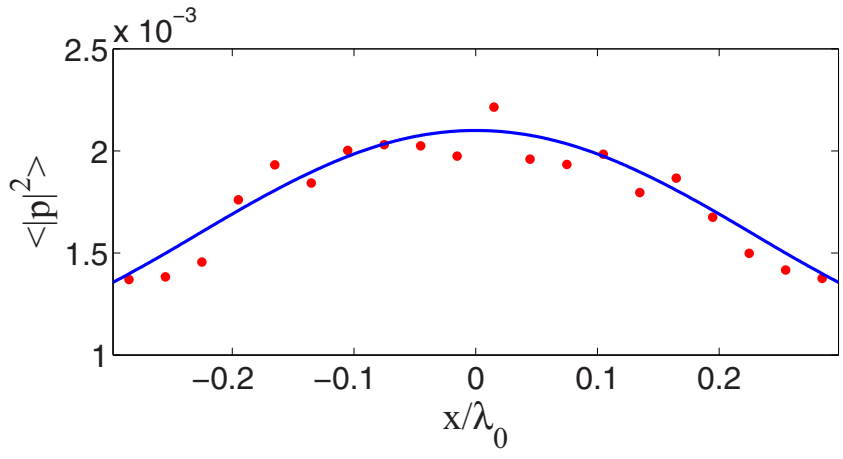

FIG. 8. Transverse profile of the polaritonic mode (a) of Fig. 2(a) (red dots) and of the macroscopic mode (blue solid curves). The profile has been obtained by averaging the dipole moments squared inside a thin slice at $z \simeq-1.3 \lambda_{0}$ and along the $y$ axis.

(microscopic) polaritonic modes with the macroscopic modes of the corresponding effective homogeneous object.

\section{ABSORPTION}

In the macroscopic model, the appearance of an imaginary part of the effective permittivity (see Fig. 6) accounts for losses, which correspond to an energy transfer from the coherent field to the incoherent (diffuse) field. Here, we demonstrate that these quantities are actually equivalent. First, we discuss the microscopic system. From an energy balance, it can be shown that the total scattered power leading to extinction $P_{\mathrm{ext}}^{\text {micro }}=P_{\mathrm{s}, \mathrm{coh}}^{\text {micro }}+P_{\mathrm{s}, \text { incoh }}^{\text {micro }}$, where $P_{\mathrm{s}, \mathrm{coh}}^{\text {micro }}$ is the coherent scattered power and $P_{\mathrm{s} \text {,incoh }}^{\text {micro }}$ the incoherent scattered power [50]. As we assume elastic light scattering, there is no absorption in the microscopic system. In the macroscopic system, there is both absorption and light scattering: $P_{\mathrm{ext}}^{\text {macro }}=P_{\mathrm{s}}^{\text {macro }}+$ $P_{\text {abs }}^{\text {macro }}$. As the ensemble-averaged electric field follows the Helmholtz equation with an effective permittivity, $P_{\mathrm{s}, \mathrm{coh}}^{\text {micro }}=$ $P_{\mathrm{s}}^{\text {macro }}$. Because of the equivalence between the microscopic and macroscopic systems, their extinction is the same. From this simple reasoning, it follows that $P_{\mathrm{s}, \text { incoh }}^{\text {micro }}=P_{\mathrm{abs}}^{\text {macro }}$. While losses are generally considered as being irreversible as a result of dephasing processes, e.g., coupling to phonons, we note that this cannot be the case here, as there is no loss mechanism in the microscopic model. Before averaging, the field scattered by a single realization of the cloud can be time reversed, provided that the scatterers' positions have not changed. After averaging, only the coherent field has a well-defined phase and can be time reversed. In summary, in the presence of dephasing processes, or when the positions of the atoms are changed randomly from one realization to another (as is the case when we ensemble average), the coupling of the incident light to dipole fluctuations leads to an irreversible radiation of incoherent light.

\section{CONCLUSION}

In conclusion, we have shown the existence of polaritonic modes in a dense atomic system. These polaritonic modes do not depend on the atomic positions but only on the shape and size of the cloud and on the atomic density; they are spatially delocalized and strongly superradiant. We have shown that they can be identified to the macroscopic modes of a homogeneous object with an effective permittivity. These results apply not only to cold atomic clouds but also to any dense system of resonant scatterers such as molecules or quantum dots. Our work provides a unified vision of scattering by dense systems of resonant scatterers.

\section{ACKNOWLEDGMENTS}

We thank V. Sandoghdar and R. Carminati for fruitful discussions. We acknowledge support from the E.U. through the ERC Starting Grant ARENA, Triangle de la Physique (COLISCINA project), the French National Research Agency (ANR) as part of the "Investissements d'Avenir" program (Labex PALM, ANR-10-LABX-0039), and Region Ile-deFrance (LISCOLEM project). N.J.S. is supported by Triangle de la Physique and Université Paris-Sud. J.-J.G. acknowledges support from Institut Universitaire de France.

\section{APPENDIX: COMPARING MICROSCOPIC AND MACROSCOPIC MODES}

As can be seen from Fig. 7 in the main text, the macroscopic modes coincide very well with the microscopic polaritonic modes. In this appendix, we detail the procedure used to compare the macroscopic mode with the average dipole moment square.

Let us note that the dipole moment square for a single realization [see Fig. 4(a) of the main text] corresponds to a single eigenmode. In the main text, the procedure of obtaining these eigenmodes has been explained. From the derivation, it follows that these modes are calculated in the absence of an external driving field, so the dipole moments are known apart from a multiplication factor. This multiplication factor is obtained by normalizing the modes, which has been done as explained in the main text by imposing $\sum_{j}\left|\mathbf{p}_{j}^{\beta}\right|^{2}=1$.

Obviously, the normalization issue also arises for the macroscopic modes. The latter have been normalized such that their intensity coincides well with the average dipole moment square. This way of comparing macroscopic and microscopic modes is consistent with the fact that $\mathbf{p} \propto \mathbf{E}$. Lastly, we note that $\left\langle|\mathbf{p}|^{2}\right\rangle=|\langle\mathbf{p}\rangle|^{2}+\left\langle|\delta \mathbf{p}|^{2}\right\rangle$. As a consequence, $\left\langle|\mathbf{p}|^{2}\right\rangle$ exhibits an offset, which is due to dipole moment fluctuations. This explains the fact that a significant offset as for $\left\langle|\mathbf{p}|^{2}\right\rangle$ is not present for $|\mathbf{E}|^{2}$. In order to superimpose them, we have added a constant offset to the macroscopic $|\mathbf{E}|^{2}$.
[1] C. F. Bohren and D. R. Huffman, Absorption and Scattering of Light by Small Particles (John Wiley \& Sons, New York, 2008).
[2] U. Frisch, in Probabilistic Methods in Applied Mathematics, edited by A. A. Bharuch-Reid (Academic Press, New York, 1968), Vols. I and II. 
[3] P. Sheng, Introduction to Wave Scattering, Localization, and Mesoscopic Phenomena (Academic Press, New York, 1995).

[4] A. Lagendijk and B. van Tiggelen, Resonant multiple scattering of light, Phys. Rep. 270, 143 (1996).

[5] S. Durant, O. Calvo-Perez, N. Vukadinovic, and J.-J. Greffet, Light scattering by a random distribution of particles embedded in absorbing media: Diagrammatic expansion of the extinction coefficient, J. Opt. Soc. Am. A 24, 9 (2007).

[6] P. Mallet, C. A. Guérin, and A. Sentenac, Maxwell-Garnett mixing rule in the presence of multiple scattering: Derivation and accuracy, Phys. Rev. B 72, 014205 (2005).

[7] J. C. Maxwell-Garnett, Colors in metal glasses and in metallic films, Philos. Trans. R. Soc. A 203, 385 (1904).

[8] D. A. G. Bruggeman, Berechnung verschiedener physikalischer Konstanten von heterogenen Substanzen. I. Dielektrizitätskonstanten und Leitfähigkeiten der Mischkörper aus isotropen Substanzen, Ann. Phys. 416, 636 (1935).

[9] G. Labeyrie, F. de Tomasi, J.-C. Bernard, C. A. Müller, C. Miniatura, and R. Kaiser, Coherent Backscattering of Light by Cold Atoms, Phys. Rev. Lett. 83, 5266 (1999).

[10] D. Wilkowski, Y. Bidel, T. Chaneliére, D. Delande, T. Jonckheere, B. Klappauf, G. Labeyrie, Ch. Miniatura, C. A. Müller, O. Sigwarth, and R. Kaiser, Coherent backscattering of light by resonant atomic dipole transitions, J. Opt. Soc. Am. B 21, 183 (2004).

[11] G. Labeyrie, D. Delande, C. A. Müller, C. Miniatura, and R. Kaiser, Multiple scattering of light in a resonant medium, Opt. Commun. 243, 157 (2004).

[12] J. Pellegrino, R. Bourgain, S. Jennewein, Y. R. P. Sortais, A. Browaeys, S. D. Jenkins, and J. Ruostekoski, Observation of Suppression of Light Scattering Induced by Dipole-Dipole Interactions in a Cold-Atom Ensemble, Phys. Rev. Lett. 113, 133602 (2014).

[13] O. Morice, Y. Castin, and J. Dalibard, Refractive index of a dilute Bose gas, Phys. Rev. A 51, 3896 (1995).

[14] J. Ruostekoski and J. Javanainen, Quantum field theory of cooperative atom response: Low light intensity, Phys. Rev. A 55, 513 (1997).

[15] J. Javanainen, J. Ruostekoski, Y. Li, and S.-M. Yoo, Shifts of a Resonance Line in a Dense Atomic Sample, Phys. Rev. Lett. 112, 113603 (2014).

[16] Y. A. Fofanov, A. S. Kuraptsev, I. M. Sokolov, and M. D. Havey, Dispersion of the dielectric permittivity of dense and cold atomic gases, Phys. Rev. A 84, 053811 (2011).

[17] S. Balik, A. L. Win, M. D. Havey, I. M. Sokolov, and D. V. Kupriyanov, Near-resonance light scattering from a high-density ultracold atomic ${ }^{87}$ Rb gas, Phys. Rev. A 87, 053817 (2013).

[18] K. Kemp, S. J. Roof, M. D. Havey, I. M. Sokolov, and D. V. Kupriyanov, Cooperatively enhanced light transmission in cold atomic matter, arXiv:1410.2497.

[19] L. Chomaz, L. Corman, T. Yefsah, R. Desbuquois, and J. Dalibard, Absorption imaging of a quasi-two-dimensional gas: A multiple scattering analysis, New J. Phys. 14, 055001 (2012).

[20] L. Bellando, A. Gero, E. Akkermans, and R. Kaiser, Cooperative effects and disorder: A scaling analysis of the spectrum of the effective atomic Hamiltonian, Phys. Rev. A 90, 063822 (2014).

[21] R. A. de Oliveira, M. S. Mendes, W. S. Martins, P. L. Saldanha, J. W. R. Tabosa, and D. Felinto, Single-photon superradiance in cold atoms, Phys. Rev. A 90, 023848 (2014).
[22] P. Tighineanu, R. S. Daveau, T. B. Lehmann, H. E. Beere, D. A. Ritchie, P. Lodahl, and S. Stobbe, Single-Photon Superradiance from a Quantum Dot, Phys. Rev. Lett. 116, 163604 (2016).

[23] M. O. Araújo, I. Krešić, R. Kaiser, and W. Guerin, Superradiance in a large cloud of cold atoms in the linear-optics regime, arXiv:1603.07204.

[24] S. J. Roof, K. J. Kemp, M. D. Havey, and I. M. Sokolov, Observation of single-photon superradiance and the cooperative Lamb shift in an extended sample of cold atoms, arXiv:1603.07268.

[25] T. Bienaimé, R. Bachelard, N. Piovella, and R. Kaiser, Cooperativity in light scattering by cold atoms, Fortschr. Phys. 61, 377 (2013).

[26] Y. Li, J. Evers, W. Feng, and S.-Y. Zhu, Spectrum of collective spontaneous emission beyond the rotating-wave approximation, Phys. Rev. A 87, 053837 (2013).

[27] I. M. Sokolov, M. D. Kupriyanova, D. V. Kupriyanov, and M. D. Havey, Light scattering from a dense and ultracold atomic gas, Phys. Rev. A 79, 053405 (2009).

[28] A. A. Svidzinsky, J.-T. Chang, and M. O. Scully, Cooperative spontaneous emission of $N$ atoms: Many-body eigenstates, the effect of virtual Lamb shift processes, and analogy with radiation of $N$ classical oscillators, Phys. Rev. A 81, 053821 (2010).

[29] S. E. Skipetrov and A. Goetschy, Eigenvalue distributions of large Euclidean random matrices for waves in random media, J. Phys. A: Math. Theor. 44, 065102 (2011).

[30] A. Goetschy and S. E. Skipetrov, Non-Hermitian Euclidean random matrix theory, Phys. Rev. E 84, 011150 (2011).

[31] A. Goetschy and S. E. Skipetrov, Euclidean matrix theory of random lasing in a cloud of cold atoms, Europhys. Lett. 96, 34005 (2011).

[32] J. J. Hopfield, Theory of the contribution of excitons to the complex dielectric constant of crystals, Phys. Rev. 112, 1555 (1958).

[33] A. S. Davydov, in Theory of Solids (Nauka, Moscow, 1980).

[34] J. Bellessa, C. Bonnand, J. C. Plenet, and J. Mugnier, Strong Coupling between Surface Plasmons and Excitons in an Organic Semiconductor, Phys. Rev. Lett. 93, 036404 (2004).

[35] J. Dintinger, S. Klein, F. Bustos, W. L. Barnes, and T. W. Ebbesen, Strong coupling between surface plasmon-polaritons and organic molecules in subwavelength hole arrays, Phys. Rev. B 71, 035424 (2005)

[36] T. K. Hakala, J. J. Toppari, A. Kuzyk, M. Pettersson, H. Tikkanen, H. Kunttu, and P. Törmä, Vacuum Rabi Splitting and Strong-Coupling Dynamics for Surface-Plasmon Polaritons and Rhodamine 6G Molecules, Phys. Rev. Lett. 103, 053602 (2009).

[37] S. Aberra Guebrou, C. Symonds, E. Homeyer, J. C. Plenet, Yu. N. Gartstein, V. M. Agranovich, and J. Bellessa, Coherent Emission from a Disordered Organic Semiconductor Induced by Strong Coupling with Surface Plasmons, Phys. Rev. Lett. 108, 066401 (2012).

[38] D. E. Gómez, K. C. Vernon, P. Mulvaney, and T. J. Davis, Surface plasmon-mediated strong exciton-photon coupling in semiconductor nanocrystals, Nano Lett. 10, 274 (2010).

[39] L. Shi, T. K. Hakala, H. T. Rekola, J.-P. Martikainen, R. J. Moerland, and P. Törmä, Spatial Coherence Properties of Organic Molecules Coupled to Plasmonic Surface Lattice Resonances in the Weak and Strong Coupling Regimes, Phys. Rev. Lett. 112, 153002 (2014). 
[40] H. Fidder, J. Knoester, and D. A. Wiersma, Superradiant emission and optical dephasing in J-aggregates, Chem. Phys. Lett. 171, 529 (1990).

[41] D. E. Gómez, S. S. Lo, T. J. Davis, and G. V. Hartland, Picosecond kinetics of strongly coupled excitons and surface plasmon polaritons, J. Phys. Chem. B 117, 4340 (2012).

[42] L. Novotny and B. Hecht, in Principles of Nano-optics (Cambridge University Press, Cambridge, UK, 2006).

[43] Due to the symmetry of the average atomic sample, polaritonic modes are actually found in pairs with very close eigenfrequencies for each realization.

[44] S. Jennewein, M. Besbes, N. J. Schilder, S. D. Jenkins, C. Sauvan, J. Ruostekoski, J.-J. Greffet, Y. R. P. Sortais, and A. Browaeys, Coherent scattering of near-resonant light by a dense microscopic cold atomic cloud, Phys Rev. Lett. 116, 233601 (2016).
[45] N. W. Ashcroft and N. D. Mermin, in Solid State Physics (Holt, Reinhart, and Winston, New York, 1976).

[46] J. Javanainen and J. Ruostekoski, Light propagation beyond the mean-field theory of standard optics, Opt. Express 24, 993 (2016).

[47] E. Silberstein, P. Lalanne, J.-P. Hugonin, and Q. Cao, Use of grating theories in integrated optics, J. Opt. Soc. Am. A 18, 2865 (2001).

[48] R. Pierrat and R. Carminati, Spontaneous decay rate of a dipole emitter in a strongly scattering disordered environment, Phys. Rev. A 81, 063802 (2010).

[49] Q. Bai, M. Perrin, C. Sauvan, J.-P. Hugonin, and P. Lalanne, Efficient and intuitive method for the analysis of light scattering by a resonant nanostructure, Opt. Express 21, 27371 (2013).

[50] M. Born and E. Wolf, Principles of Optics, 7th ed. (Cambridge University Press, Cambridge, UK, 1999). 